\title{
Conformational Aspects of Polypeptides Containing Ionizable Side Chains
}

\author{
Seiji Tanaka and Akio Nakajima* \\ Department of Polymer Chemistry, Kyoto University, \\ Sakyo-ku, Kyoto, Japan. \\ (Received April 15, 1970)
}

\begin{abstract}
Conformational analyses for polypeptides having ionizable side chains were carried out by varying side-chain length. The entropy of the chain in the randomcoil state was evaluated from the calculated conformational energy. For such acidic polypeptides as poly(L-aspartic acid) and poly(L-glutamic acid), the helix conformation in an ionized state is more stable than in an un-ionized state. On the other hand, with basic polypeptides having long side chains, such as poly(L-lysine), the helix conformation is destabilized by ionization of the side chains. These theoretical results successfully interpret results obtained experimentally on the $\mathrm{pH}$-induced transition of polypeptides.

KEY WORDS Polymer / Polypeptide / Polyelectrolyte / Solution /

Conformation Energy, Entropy / Statistics of Chain Molecule /

pH-induced Transition /
\end{abstract}

Helix-coil transition in polypeptide chains which contain ionizable side chains has been investigated by many authors experimentally ${ }^{1-4}$. For example, Doty and his coworkers ${ }^{1}$ found that the conformation of poly(L-glutamic acid) depends on $\mathrm{pH}$ of the solution, i.e., in $0.2-M$ $\mathrm{NaCl}$ dioxane solution, the polypeptide chains are in helical conformation at low $\mathrm{pH}$, but are in random-coil conformation at high $\mathrm{pH}$. Thus the conformation of such polypeptides as poly(glutamic acid), poly(aspartic acid) and polylysine, containing ionizable side chains, is known to be a function of the degree of ionization. At low $\mathrm{pH}$, where the carboxyl groups are in un-ionized form $(-\mathrm{COOH})$, the chains are in helical conformation, probably in the $\alpha$-helix form. At high $\mathrm{pH}$, where ionization of side chains occurs and the side chain groups are therefore in charged form $\left(\mathrm{COO}^{-}\right)$, the chains are in randomcoil conformation. Doty, et al. ${ }^{1}$, have suggested that the helical conformation cannot withstand the electrostatic repulsion between the charged groups of the side chains. Applequist and Doty ${ }^{4}$ also found helix-coil transition induced by $\mathrm{pH}$ variation for poly(L-lysine)(PLL). Further, Myer $^{5}$, Tiffany, et al. ${ }^{6}$, and Ciferri, et al. ${ }^{7}$, have investigated the $\mathrm{pH}$-induced helix-coil transi-

* To whom correspondence should be addressed. tion of PLL and poly(L-glutamic acid)(PLGA) by circular dichroism spectral ${ }^{5,6}$, and potentiometric titration measurements ${ }^{7}$. According to Zimm and Rice, in the case of PLGA, the distances between the charge in a $\mathrm{COO}^{-}$group and those in its four neighboring groups, in helical form, are 10.1, 13.1, 7.9, and $7.5 \AA$, while those in random-coil form, are $c a$. 10, ca. $10, c a .10$ and $c a .16 \AA$. They therefore concluded that the electrostatic interactions of side chains in an ionized state must stabilize the random-coil form, and from this developed a statistical mechanical treatment of the helixcoil transition of ionizable polypeptides.

Recently, Krimm and Mark $^{9}$ discussed the effects of interactions among the charged side chains in the chain conformation of polypeptides. They took into consideration both van der Waals interactions between all pairs of nonbonded atoms and long-range electrostatic interactions, for each dipeptide unit along the chain, by assuming trans conformation for all the amide bonds. If the ionic charges on the side chains are screened by added electrolytes, the long-range charge interactions may be reduced and the short-range interactions become important. Consequently, so far as interactions between side chain charges are concerned, the 


\section{S. TANAKA and A. NAKAJIMA}

interactions between side chains attached to the neighboring residues become important. In this work, we will evaluate the conformation energy and entropy according to the dipeptide model with side chains of different lengths, leaving aside interactions between neighboring side-chain charges, and discuss the effect of side-chain length on the $\mathrm{pH}$-induced helix-coil transition for polypeptides with acidically or basically ionizable side chains. When the interactions between side-chain charges of neighboring residues are not taken into consideration, the conformation may correspond to just the early stage of the $\mathrm{pH}$-induced helix - coil transition, i.e., the stage of the low degree of ionization. To this end, we have taken into account the following kinds of interactions within the dipeptide unit to estimate the chain conformation of ionized polypeptides.

(i) Tortional energy, $V_{(\mathrm{T})}(\varphi)$ and $V_{(\mathrm{T})}(\psi)$, about the bonds, $\mathrm{N}-\mathrm{C}^{\alpha}$ and $\mathrm{C}^{\alpha}-\mathrm{C}^{\prime}$

(ii) The sum of the energies of nonbonded atom interactions due to the dispersion and repulsion forces, $V_{(\mathrm{AA})}$

(iii) Energy of interaction between neighboring amide dipoles, $V_{(\mathrm{DD})}$

(iv) The sum of the electrostatic interactions between the amide dipole and the side chain ion, $V_{\text {(DI) }}$

(v) The sum of the energies of the induced interactions between the side-chain ion and each of the backbone atoms, $V_{(\mathrm{AI})}$

In addition, when the polypeptide is in helix conformation the energy due to intramolecular hydrogen bonds should be added.

The chain conformation of polyamino acids with nonionizable side chains has been investigated by taking into account the interactions (i)-(iii) by many researchers ${ }^{11-16}$. In this work, we will discuss the effects of interactions covered from (i) to (v) on the chain conformation by using the dipeptide model, in which, however, long-range interactions between side-chain charges are not included.

\section{MOLECULAR MODEL AND POTENTIAL FUNCTION}

\section{Molecular Model}

A section of a $\alpha$-L-polypeptide chain with ionizable side chains in its fully extended form is illustrated in Figure $1 \mathrm{a}$. The side chain $\mathrm{R}$ is indicated by $\mathrm{R}=-\left(\mathrm{CH}_{2}\right)_{x}-\mathrm{COOH}$ for polyacids and by $\mathrm{R}=-\left(\mathrm{CH}_{2}\right)_{x}-\mathrm{NH}_{2}$ for polybases with $x=0,1,2,3,4,5$, and 6 . For example, $\mathrm{R}=-\mathrm{CH}_{2}-\mathrm{COOH}(x=1)$ and $\mathrm{R}=-\left(\mathrm{CH}_{2}\right)_{2}-$ $\mathrm{COOH}(x=2)$ respectively correspond to poly(L-aspartic acid) (PLAA) and poly(L-glutamic acid) (PLGA), and $\mathrm{R}=-\left(\mathrm{CH}_{2}\right)_{4}-\mathrm{NH}_{2}$ corresponds to poly-L-lysine (PLL). Strictly speaking, the conformation of side chains may depend on that of the backbone chain. In order to simplify the calculation, however, we have employed a rather simplified model as used by Krimm and Mark ${ }^{9}$, which assumes that the three rotational states (trans, gauche, and another gauche) about each $\mathrm{C}-\mathrm{C}$ bond in the side chain occur with equal probability, and that the ionic charge of the side chain is located at a point obtained by projecting the side chain in the $\mathrm{C}^{\alpha}-\mathrm{C}^{\beta}$ direction. In Figure $1 \mathrm{a}$, point $\mathrm{I}$ indicates the location of the ionic charge, which is a function of the number of the $\mathrm{CH}_{2}$ groups in the side chain. For the ionized carboxyl group, $-\mathrm{COO}^{-}$, of the acidic side chain, $\mathrm{C}_{x}, \mathrm{C}, \mathrm{O}^{\prime}$, and $\mathrm{O}^{\prime \prime}$ atoms (see Figure $1 \mathrm{~b}$ ) were assumed to be coplanar with the bond angle of $\mathrm{O}^{\prime \prime}-\mathrm{C}-\mathrm{O}^{\prime}$ assigned the value $125^{\circ}$, and bond length of $\mathrm{C}-\mathrm{O}$ equal to $1.27 \AA$. The negative charge was assumed, according to Pauling ${ }^{10}$, to be located at the middle point of the line connecting the $\mathrm{O}^{\prime}$ and $\mathrm{O}^{\prime \prime}$ atoms (this assignment is somewhat different from the Krimm and Mark's ${ }^{9}$ treatment which


(a)

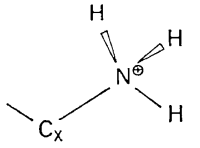

(b)

Figure 1. Geometry of the L-polypeptide with ionized side chain. 
located the charge on the $\mathrm{C}$ atom of the $-\mathrm{COO}^{-}$group). Thus the distance between the $\mathrm{C}_{x}$ atom and the ion in our treatment is equal to $2.11 \AA$. On the other hand, for the $-\mathrm{NH}_{3}{ }^{+}$group of the basic side chain, the positive charge was assumed ${ }^{10}$ to be located on the $\mathrm{N}$ atom. Accordingly, the charge distances from the $\mathrm{C}^{\alpha}$ atom to the ionic charge for side chains containing $x=0,1,2,3,4,5$, and $6 \mathrm{CH}_{2}$ groups amount to $2.11,2.24,4.15,4.28,6.28,6.32$, and $8.24 \AA$, respectively, for the acidic side chain, and $1.47,2.02,3.51,4.06,5.55,6.10$, and $7.59 \AA$, respectively, for the basic one. Other molecular parameters such as bond lengths and bond angles used in this work are the same as those normally being used ${ }^{10,11}$. The angles of rotation about the $\mathrm{N}-\mathrm{C}^{\alpha}$ and $\mathrm{C}^{\alpha}-\mathrm{C}^{\prime}$ bonds are denoted by $\varphi$ and $\psi$, to which are assigned values of zero in the planar, trans conformation. The sense of rotations, and the coordinate systems affixed to each atom of the chain skeleton are the same as our preceeding paper ${ }^{11}$. Thus, the distances between atoms are calculated by the vector sum of each bond.

\section{Potential Functions}

The chain conformation of polypeptides in solution is described as a function of rotational angles, $\varphi$ and $\psi$ about the two bonds divided by two planar trans-amide bonds, i.e., the conformational energy $V(\varphi, \psi)$ is expressed by

$$
V(\varphi, \phi)=V_{(\mathrm{T})}(\varphi)+\sum_{j, k} V_{j k}(\varphi, \phi)
$$

where $V_{(\mathrm{T})}(\varphi)$ and $V_{(\mathrm{T})}(\psi)$ denote the intrinsic three-fold torsional potentials about the bond $\mathrm{N}-\mathrm{C}^{\alpha}$ and $\mathrm{C}^{\alpha}-\mathrm{C}^{\prime}$, respectively, and $\sum V_{j k}(\varphi, \psi)$ is the sum of all types of interactions between nonbonded atoms, $j$ and $k$. For such nonionizable polypeptides as polyglycine and polyalanine, the term, $V_{j k}(\varphi, \psi)$ is given by the sum of contributions (ii), $V_{(\mathrm{AA})}$, and (iii), $V_{(\mathrm{DD})}$, mentioned earlier, and expressed by eq 2 .

$$
V_{j k}(\varphi, \phi)=V_{(\mathrm{AA}) j k}(\varphi, \phi)+V_{(\mathrm{DD}) j_{k}}(\varphi, \phi)
$$

For polypeptides with ionizable side chains, contributions (iv), $V_{(\mathrm{DI})}$, and (v), $V_{(\mathrm{AI})}$, should be added to contributions (ii) and (iii), resulting in the following equations.

$$
\begin{aligned}
V_{j k}(\varphi, \psi)= & V_{(\mathrm{AA}) j_{k}}(\varphi, \psi)+V_{(\mathrm{DD}) j j_{k}}(\varphi, \psi) \\
& +V_{(\mathrm{DI}) j k}(\varphi, \psi)+V_{(\mathrm{AI})}(\varphi, \psi)
\end{aligned}
$$

Further consideration will now be given to the potential functions of these different contributions.

The torsional potential functions, $V_{(\mathrm{T})}(\varphi)$ and $V_{(\mathrm{T})}(\psi)$ used were the three-fold potential functions employed by Brant and Flory ${ }^{13}$. The numerical values of the torsional barrier heights used were also the same as those used by Brant and Flory ${ }^{13}$.

For the London dispersion and repulsion energy, $V_{(\mathrm{AA}) j k}(\varphi, \psi)$ between nonbonded atoms, $j$ and $k$, the Lennard-Jones-type "6-12" potential function was used as a function of the separation $r_{j k}$ of their centers. The numerical values of the atomic polarizability and the effective number of electrons in the outer electronic subshell, used for estimating the parameters in attractive terms of the "6-12" type equation, were cited from the paper by Brant and Flory ${ }^{13}$. For obtaining the parameter in the -12 th power term, the sum of the van der Waals radii of two atoms was assumed to be the minimum atomic distance, $r_{\min }$. This point differs from Brant's treatment ${ }^{12}$.

The mutual potential energy, $V_{(\mathrm{DD})}$, of the two amide dipoles in the backbone chain was calculated by the "point monopole" approximation $^{12,14,15}$, after distributing the corresponding charge on each atom comprising the amide groups. In our treatment, the interaction between the two amide dipoles is given by the sum of the Coulombic interactions between these atomic charges. This approximation has been shown, by Brant, et al. ${ }^{12}$, to give a characteristic ratio $\left\langle R_{0}{ }^{2}\right\rangle / n l^{2}$ closer to the experimental value than that obtained by the equation to calculate directly the interaction between two dipoles.

The interaction energy, $V_{(\mathrm{DI})}$, between the amide dipole and side chain ion was calculated as the sum of Coulombic interactions between the side chain ion and each of point monopoles mentioned above. The numerical value of the partial charge assigned to each atom of the amide group was taken from the paper by Scott and $\mathrm{Scheraga}^{14}$. For the $-\mathrm{COO}^{-}$or $-\mathrm{NH}_{3}^{+}$ group, the ionic charge is $-e$ or $+e$, where $e$ denotes the elementary charge, because the number of effective charge $z$ is 1 . For calcu- 


\section{S. TANAKA and A. Nakajima}

lations of both $V_{(\mathrm{DD})}$ and $V_{(\mathrm{DI})}$, the effective dielectric constant, $\varepsilon$, was taken as $\varepsilon=3.5$, in accordance with Brant, et al. ${ }^{13}$

Lastly, we will describe the last term, $V_{(\mathbf{A I}) j k}(\varphi, \psi)$, in eq 3 , which originates from the induced interaction between the side chain ion and each of the backbone atoms. When an electric field with electric intensity $E$ is applied to an atom having excess polarizability $\alpha$, an electric moment amounting to $\alpha E$ will be induced in the atom. When one atom or one ion is placed in the electric field of another ion, the electric field formed by the latter ion is expressed by $z e / r^{2}$, where $r$ denotes the distance between two ions or between the ion and atom. The first atom or ion is, then, polarized by the electric field formed by the second ion, and induces an electric moment of $\alpha\left(z e / r^{2}\right)$. The dipole thus produced may affect the first ion, and may attract the second ion with a force of $-2 z^{2} e^{2} / r^{5}$. This force is always an attraction force ${ }^{16}$ and therefore, the potential energy is given by eq 4 .

$$
V_{(\mathrm{AI}) j k}=-\frac{\alpha z^{2} e^{2}}{2 r^{4}}
$$

(Eq 4 was derived by ignoring the effect of dielectric constant.) Such potential as given by eq 4 originates from electrostatic induction (polarization), and must be distinguished from the polarization by instantaneous dipoles due

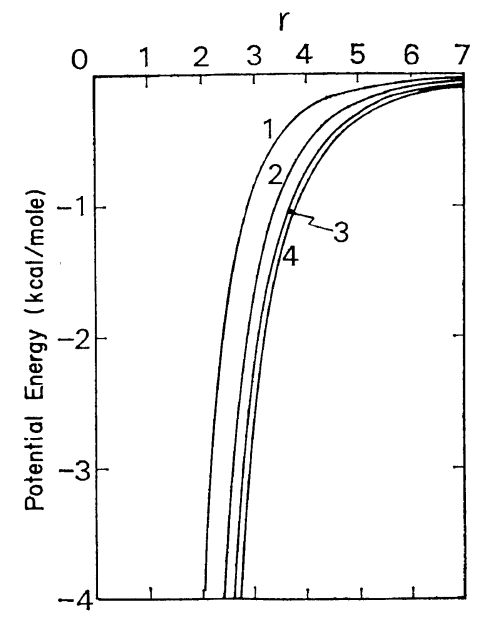

Figure 2. Plots of the induced electrostatic energy vs. the distance $r(\AA)$ between the ion and the atom $\mathrm{H}^{\prime}$ (curve 1), $\mathrm{O}$ (curve 2), $\mathrm{N}$ (curve 3 ), or $\mathrm{C}^{\prime}$ (curve 4).
Table I. Partial charge, $\delta_{j}$, and polarizability coefficient, $\alpha_{j}$, for atom $j$ composing amide group

\begin{tabular}{lcc}
\hline Atom & $\begin{array}{c}\delta_{j}(\text { in unit of } \\
\text { elementary charge } e)^{\mathrm{a}}\end{array}$ & $\alpha_{j}\left(\AA^{3}\right)^{\mathrm{b}}$ \\
\hline $\mathrm{H}^{\prime}$ & 0.272 & 0.42 \\
$\mathrm{~N}$ & -0.305 & 1.15 \\
$\mathrm{C}^{\prime}$ & 0.449 & 1.30 \\
$\mathrm{O}$ & -0.416 & 0.84 \\
\hline
\end{tabular}

a Ref 9. ${ }^{b}$ Ref 6.

to the London force.

For the polypeptides discussed here, the ionic charge is on the side chain attached to the $\alpha$ carbon atom. Therefore the interactions between this ionic charge and each of the atoms comprising the two amide groups connected by this $\alpha$-carbon atom can be regarded as important in understanding the backbone chain conformation.

Figure 2 shows the induced electrostatic energy as a function of the distance $r$ between the ion and atoms, calculated by eq 4 using the polarizability values for such atoms as $\mathrm{H}, \mathrm{O}, \mathrm{N}$, and $\mathrm{C}$ reported by Brant and Flory $^{13}$. As seen in Figure 2, this force can be regarded as a relatively short-range force in nature. Curves 1, 2, 3, and 4 represent the energies between the ion and, respectively, $\mathrm{H}^{\prime}, \mathrm{O}, \mathrm{N}$, and $\mathrm{C}^{\prime}$ atoms. It should also be pointed out that this energy is always negative. Therefore, the added contribution of $V_{(\mathrm{AI}) j k}$ and $V_{(\mathrm{DI}) j k}$ to the total energy may be largely affected by whether $V_{(\mathrm{DI}) j k}$ is repulsive (positive) or attractive (negative). Thus the contribution of this energy $V_{(A \mathrm{I}) j / c}$ to total conformational energy should not be neglected. The effect of dielectric constant for $V_{(A I) j k}$ may be ignored due to its short-range nature.

The numerical values of the polarizability $\alpha\left(\AA^{3}\right)$ and partial charge $\delta$ for each atom comprising the amide group are listed in Table I.

\section{RESULTS AND DISCUSSION}

\section{Conformational Energy}

The conformational energy of polypeptides with ionizable side chains is calculated from eq 1 and 3. We will, initially, deal with the contribution from the London interaction 


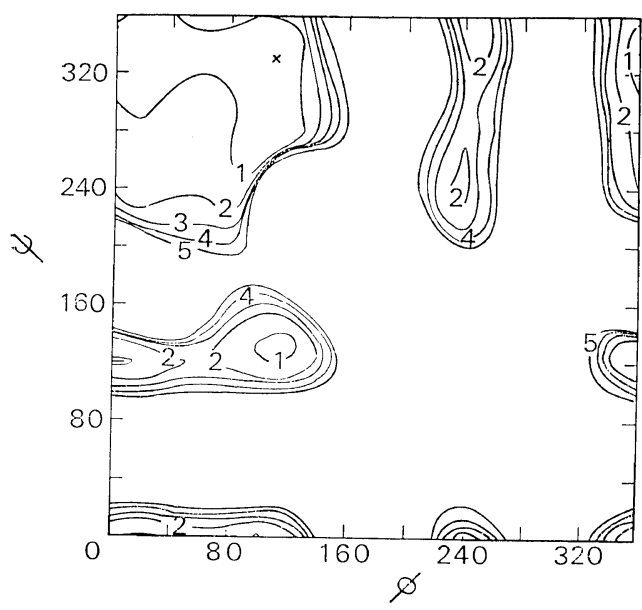

Figure 3. Contour map of total conformational energy at $1 \mathrm{kcal} / \mathrm{mol}$ interval for poly(L-alanine). The lowest energy point (the absolute energy value is $-2.22 \mathrm{kcal} / \mathrm{mol}$ ) is indicated by $\times$.

$V_{(\mathrm{AA}) j k}$. According to Scheraga, et al..$^{17,18}$, and Ramachandran, et al. ${ }^{19}$, the London energy maps of polypeptides with linear side chains longer than that of polyalanine $(x=1)$ do not differ significantly from that of polyalanine. We start with the calculation of the contour maps for polyalanine and polyglycine from eq 1 and 2 and then assume that the result obtained for polyalanine is the same as that of $\alpha$-L-polypeptides with longer side chains. The energy contour map for polyalanine calculated, at intervals of $10^{\circ}$ in $\varphi$ and $\psi$, from eq 1 and 2 is shown in Figure 3. In Figure 3, the lowest energy point appears at $\varphi=100^{\circ}$ and $\psi=330^{\circ}$, and its absolute value is $-2.22 \mathrm{kcal} / \mathrm{mol}$. The energy contours are illustrated at intervals of $1 \mathrm{kcal} / \mathrm{mol}$ relative to this minimum value. This map is practically the same as that obtained by Brant, et al. ${ }^{12}$, although the values of parameters, in particular of the point monopoles $\delta_{j}$ (see Table I) and of the van der Waals radii, used in this work, are somewhat different. The conformational contour map shown in Figure 3 is assumed to be applicable not only to L-polypeptide ester and poly(L-alanine), but also to polypeptides with ionizable side chains at those un-ionized states. When we add the contribution of intramolecular hydrogen bonds to the contour map shown in Figure 3, we may expect energy minima in the regions for $\alpha$-helix con-

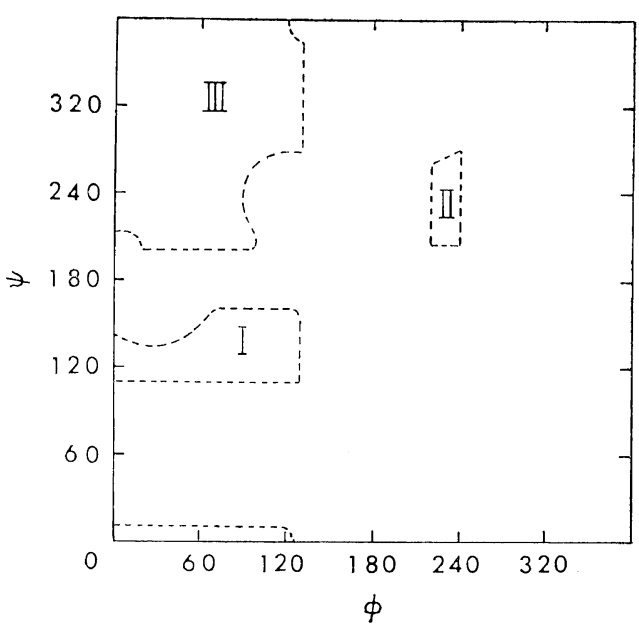

Figure 4. Steric map given by Ramachandran ${ }^{20}$ for the L-alanyl residue. Accessible domains enclosed by broken lines are designated as regions I, II, and III.

formation. Calculations for polyglycine were also carried out. Though the energy map obtained is not shown in this paper, the entropy of the random-coil form for polyglycine shown in Table IV was obtained on the basis of these calculations.

Previously, Ramachandran, et al. ${ }^{20}$, reported a steric map (Figure 4) by using the minimum contact distance (the sum of the van der Waals radii of atoms) for each pair of atoms, and pointed out that this figure adequately explained the conformations of various synthetic polypeptides and proteins derived from X-ray analysis. The portions surrounded by the broken lines in Figure 4 denote the regions obtained for the outer limit of the contact distances, with $\angle \mathrm{NC}^{\alpha} \mathrm{C}^{\prime}=110^{\circ}$. We now designate regions $\mathrm{I}$, II, and III, with the following explanation. In regard to region II, we assign to $\psi$ a wider range, $200<\psi \leq 360^{\circ}$, than that shown in Figure 4. In region $I$ are included the right-handed $\alpha$-helix and the $\pi$-helix, in II the left-handed $\alpha$-helix and the $\gamma$-helix, and in III the $\beta$-conformation and others. Brant, et $a .^{12}$, have shown, for the polyalanine model chain, that the energy minimum appeared in region I when the dipole-dipole interactions between amide groups were ignored, but moved to region III when those interactions were included. In 


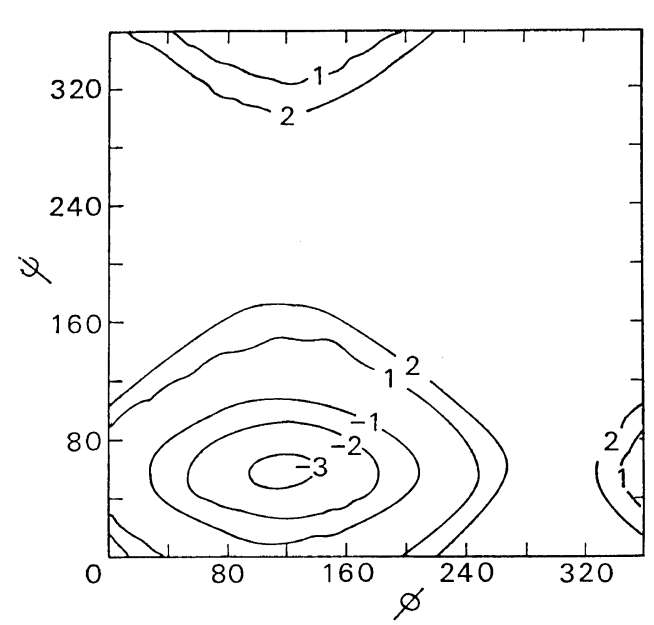

Figure 5. Contour map of electrostatic interaction energy $V_{(\mathrm{DI})}(\varphi, \phi)$ for polypeptide with acidic side chains, each of which has $x=1 \mathrm{CH}_{2}$ group (i.e., poly(L-aspartic acid)) with $\varepsilon=3.5$.

the present case (Figure 3), the energy minimum is located in region III.

We now discuss the conformational energy for polypeptides with ionized side chains. To this end, the contributions relating $V_{(D I)}$ and $V_{\text {(AI) }}$ should be added to the sum of eq 1 and 2. These two terms, $V_{(\mathrm{DI})}$ and $V_{(\mathrm{AI})}$, are not only the functions of $\varphi$ and $\phi$, but also of the charge distance, i.e., the number $x$ of $-\mathrm{CH}_{2}-$ groups in the side chain, and, moreover, depend on the sign of the ionic charge. As mentioned before, it should be noted that $V_{(\mathrm{AI}) j_{k}}(\varphi, \psi)$ is always negative (attraction force) and independent of the signs of the ionic charge. The electrostatic interaction energy map for a polypeptide with acidic side chains of $x=1$ (i.e., poly(L-aspartic acid)) is shown in Figure 5. As can be seen from Figure 5, there is minimum near $\varphi=120^{\circ}$ and $\phi=60^{\circ}$. This means that the contribution from the electrostatic interaction, $V_{(\mathrm{DI}) j k}(\varphi, \psi)$, in this case may affect the association with region I, namely the right-handed $\alpha$-helix conformation for example, but not with the region II, namely the left-handed $\alpha$-helix conformation.

Though not shown here, the contour map for polypeptides with $-\left(\mathrm{CH}_{2}\right)_{x}-\mathrm{N}^{+} \mathrm{H}_{3}$-type side chains was approximately symmetrical with that shown in Figure 5 about the diagonal connect- ing the points $\varphi=360^{\circ}$ and $\psi=360^{\circ}$. Accordingly, the minimum was located at around $\varphi=300^{\circ}$ and $\psi=240^{\circ}$, but the absolute value of this potential energy is slightly different from that of polypeptides with acidic side chain because of the difference in the molecular model for acid and basic side chains, as noted in the previous sections. This means that the electrostatic interaction may affect the association with the region II in this case. The energy levels of the regions III and I are estimated to be more than $5 \mathrm{kcal} / \mathrm{mol}$ for small $x$.

The induced electrostatic energy, $V_{(\mathrm{AI})}$, for polypeptides with $-\left(\mathrm{CH}_{2}\right)_{x}-\mathrm{COO}^{-}$-type side chains, calculated on the basis of the potential functions as given in Figure 2, is shown in Figure 6 (for $x=1$ ). For polypeptides with $-\left(\mathrm{CH}_{2}\right)_{x}-\mathrm{NH}_{3}{ }^{+}$-type side chains, a contour map of the same type as shown in Figure 6 was obtained. In this case, however, the absolute value of the energy levels is different from the former, because the charge distances are different even for $x=1$ between the cationic and anionic side chains. As seen in Figure 6 , the minimum energy is located at $\varphi=300^{\circ}$, $\psi=60^{\circ}$. Therefore, the electrostatic induction energy may contribute to a region quite different from regions I, II, and III. In particular, region III is in a energy level higher than $7 \mathrm{kcal} / \mathrm{mole}$. relative to this minimum.

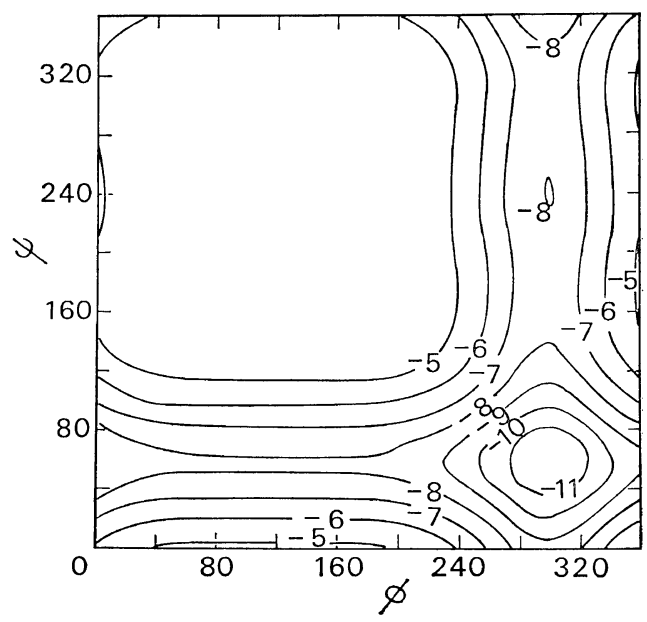

Figure 6. Contour map of induced electrostatic interaction energy $V_{(\mathrm{AI})}(\varphi, \phi)$ for poly(L-aspartic acid). 
Though the energy maps of $V_{(\mathrm{DI})}(\varphi, \phi)$ and $V_{(\mathrm{AI})}(\varphi, \phi)$ obtained for acidic and basic side chains with different $x$ are not shown here, the mean values, $\bar{V}_{\text {(DI) }}$ and $\overline{\bar{V}}_{\text {(DI) }}$ defined by eq 5 and 6 were calculated on the basis of these maps.

By exchanging the subscripts $\mathrm{D}$ and $\mathrm{A}$ appearing in eq 5 and 6 , we obtain $\bar{V}_{(\mathrm{AI})}$ and $\overline{\bar{V}}_{(\mathrm{AI})}$. The numerical values of $\bar{V}_{(\mathrm{DI})}, \bar{V}_{(\mathrm{AI})}, \overline{\bar{V}}_{(\mathrm{DI})}$, and $\overline{\bar{V}}_{(\mathrm{AI})}$ obtained for polypeptides with $-\left(\mathrm{CH}_{2}\right)_{x}$ - $\mathrm{COO}^{-}$-type and $-\left(\mathrm{CH}_{2}\right)_{x}-\mathrm{NH}_{3}{ }^{+}$-type side chains are listed in Tables II and III, respectively.

In practical calculations of eq 5 and 6 , the integrals were replaced by the summation at intervals of $20^{\circ}$ in $\varphi$ and $\psi$. The assumed temperature was $25^{\circ} \mathrm{C}$.

$\bar{V}_{(\mathrm{DI})}$ and $\bar{V}_{(\mathrm{AI})}$ represent the averages of the electrostatic interaction energy given in Figure 5 and of the induced electrostatic energy given in Figure 6, respectively, with respect to the whole range of $\varphi$ and $\psi$. On the other hand, $\overline{\bar{V}}_{\text {(DI) }}$ and $\overline{\bar{V}}_{(\mathrm{AI})}$ represent the fractional contributions from the electrostatic interaction energy and from the induced electrostatic energy, respectively, amongst the sum of these two contributions.

Now the total conformational energy of polypeptides having ionized side chains is obtained by adding the electrostatic interaction energy and the induced electrostatic energy to the energy given in Figure 3. Some examples of

$$
\begin{gathered}
\bar{V}_{(\mathrm{DI})}=\frac{\iint_{0}^{2 \pi} V_{(\mathrm{DI})}(\varphi, \psi) \exp \left[-V_{(\mathrm{DI})}(\varphi, \psi) / R T\right] \mathrm{d} \varphi \mathrm{d} \psi}{\iint_{0}^{2 \pi} \exp \left[-V_{(\mathrm{DI})}(\varphi, \psi) / R T\right] \mathrm{d} \varphi \mathrm{d} \psi} \\
\overline{\bar{V}}_{(\mathrm{DI})}=\frac{\iint_{0}^{2 \pi} \frac{V_{(\mathrm{DI})}(\varphi, \psi) \exp \left[-\left\{V_{(\mathrm{DI})}(\varphi, \psi)+V_{(\mathrm{AI})}(\varphi, \psi)\right\} / R T\right] \mathrm{d} \varphi \mathrm{d} \psi}{\iint_{0}^{2 \pi} \exp \left\{-\left\{V_{(\mathrm{DI})}(\varphi, \psi)+V_{(\mathrm{AI})}(\varphi, \psi)\right\} / R T\right] \mathrm{d} \varphi \mathrm{d} \psi}}{\int}
\end{gathered}
$$

Table II. Positions and energies of minima, and average energies for polypeptides

\begin{tabular}{|c|c|c|c|c|c|c|c|}
\hline & \multicolumn{7}{|c|}{$x$, number of $\mathrm{CH}_{2}$ groups in the side chain } \\
\hline & 0 & 1 & 2 & 3 & 4 & 5 & 6 \\
\hline \multicolumn{8}{|c|}{ Energy minimum of } \\
\hline Region $\mathrm{I}^{\mathrm{a}}$ & $\begin{array}{c}-6.74^{b} \\
(120,120)\end{array}$ & $\begin{array}{c}-6.20 \\
(120,120)\end{array}$ & $\begin{array}{c}-1.90 \\
(120,120)\end{array}$ & $\begin{array}{c}-1.79 \\
(120,120)\end{array}$ & $\begin{array}{c}-0.84 \\
(120.120)\end{array}$ & $\begin{array}{c}-0.81 \\
(120,120)\end{array}$ & $\begin{array}{c}-0.57 \\
(120,120)\end{array}$ \\
\hline Region II & $\begin{array}{c}-4.57 \\
(240,0)\end{array}$ & $\begin{array}{c}-3.98 \\
(240,0)\end{array}$ & $\begin{array}{c}0.11 \\
(240,0)\end{array}$ & $\begin{array}{c}0.19 \\
(240,0)\end{array}$ & $\begin{array}{c}0.55 \\
(240,0)\end{array}$ & $\begin{array}{c}0.55 \\
(240,0)\end{array}$ & $\begin{array}{c}0.41 \\
(240,0)\end{array}$ \\
\hline Region III & $\begin{array}{c}-7.03^{\mathrm{d}} \\
(100,0)\end{array}$ & $\begin{array}{c}-6.48 \\
(100,0)\end{array}$ & $\begin{array}{c}-2.30 \\
(120,340)\end{array}$ & $\begin{array}{c}-2.21 \\
(120,340)\end{array}$ & $\begin{array}{c}-1.56 \\
(120,340)\end{array}$ & $\begin{array}{c}-1.54 \\
(120,340)\end{array}$ & $\begin{array}{c}-1.42 \\
(120,340)\end{array}$ \\
\hline \multicolumn{8}{|c|}{ Average energye } \\
\hline $\bar{V}_{(\mathrm{DI})}$ & -2.53 & -2.49 & -0.64 & -0.53 & 0.50 & 0.54 & 0.87 \\
\hline $\bar{V}_{(\mathrm{AI})}$ & -12.3 & -11.3 & -1.64 & -1.47 & -0.37 & -0.34 & -0.13 \\
\hline$\overline{\bar{V}}_{(\mathrm{DI})}$ & -2.59 & -2.59 & -0.72 & -0.61 & 0.49 & 0.53 & 0.87 \\
\hline$\overline{\bar{V}}_{(\mathrm{AI})}$ & -8.85 & -8.06 & -1.52 & -1.38 & -0.36 & -0.34 & -0.13 \\
\hline
\end{tabular}
with $-\left(\mathrm{CH}_{2}\right)_{x}-\mathrm{COO}^{-}$-type side chains

a Regions I, II and III are designated in Figure 4.

b Absolute values of conformation energy are given in $\mathrm{kcal} / \mathrm{mol}$.

c The numerals in parenthesis denote $\phi$ and $\phi$ in degrees for the energy minimum.

d The solid letter signifies the lowest energy point on the contour map.

e The average energies $(\mathrm{kcal} / \mathrm{mol})$ are calculated from eq 5 and 6 . 


\section{S. TANAKA and A. NAKAJima}

Table III. Positions and energies of minima, and average energies for polypeptides with $-\left(\mathrm{CH}_{2}\right)_{x}-\mathrm{NH}_{3}{ }^{+}$-type side chains

\begin{tabular}{|c|c|c|c|c|c|c|c|}
\hline & \multicolumn{7}{|c|}{$x$, number of $\mathrm{CH}_{2}$ groups in the side chain } \\
\hline & 0 & 1 & 2 & 3 & 4 & 5 & 6 \\
\hline \multicolumn{8}{|c|}{ Energy minimum of } \\
\hline Region $\mathrm{I}^{\mathrm{a}}$ & $\begin{array}{l}-14.6 \\
(0,120)\end{array}$ & $\begin{array}{l}-11.0 \\
(0,120)\end{array}$ & $\begin{array}{l}-5.92 \\
(0,120)\end{array}$ & $\begin{array}{l}-5.06 \\
(0,120)\end{array}$ & $\begin{array}{l}-3.76 \\
(0,120)\end{array}$ & $\begin{array}{l}-3.49 \\
(0,120)\end{array}$ & $\begin{array}{l}-2.98 \\
(0,120)\end{array}$ \\
\hline Region II & $\begin{array}{l}-17.0 \\
(240,240)\end{array}$ & $\begin{array}{l}-13.7 \\
(240,240)\end{array}$ & $\begin{array}{c}-7.79 \\
(240,240)\end{array}$ & $\begin{array}{c}-6.52 \\
(240,240)\end{array}$ & $\begin{array}{l}-4.36 \\
(240,240)\end{array}$ & $\begin{array}{c}-3.85 \\
(240,240)\end{array}$ & $\begin{array}{c}-2.89 \\
(240,240)\end{array}$ \\
\hline Region III & $\begin{array}{l}-16.7 \\
(0,260)\end{array}$ & $\begin{array}{l}-13.5 \\
(0,260)\end{array}$ & $\begin{array}{l}-7.87 \\
(0,260)\end{array}$ & $\begin{array}{l}-6.68 \\
(0,260)\end{array}$ & $\begin{array}{l}-5.01 \\
(0,320)\end{array}$ & $\begin{array}{l}-4.65 \\
(0,320)\end{array}$ & $\begin{array}{l}-3.98 \\
(0,320)\end{array}$ \\
\hline \multicolumn{8}{|c|}{ Average energy } \\
\hline $\bar{V}_{(\mathrm{DI})}$ & -8.83 & -9.31 & -7.34 & -6.36 & -4.25 & -3.68 & -2.60 \\
\hline $\bar{V}_{(\mathrm{AI})}$ & -17.3 & -13.1 & -3.19 & -1.79 & -0.55 & -0.39 & -0.18 \\
\hline$\overline{\bar{V}}_{(\mathrm{DI})}$ & -8.63 & -9.52 & -7.50 & -6.49 & -4.30 & -3.72 & -2.62 \\
\hline$\overline{\bar{V}}_{(\mathrm{A} I)}$ & -12.8 & -9.05 & -2.89 & -1.86 & -0.63 & -0.44 & -0.19 \\
\hline
\end{tabular}

a Other low energy points on the contour map are $-16.9 \mathrm{kcal} / \mathrm{mol}$ at $\left(340^{\circ}, 260^{\circ}\right)$ and $\left(260^{\circ}, 260^{\circ}\right)$ for $x=0,-13.8 \mathrm{kcal} / \mathrm{mol}$ at $\left(260^{\circ}, 240^{\circ}\right)$ for $x=1$, and $-7.16 \mathrm{kcal} / \mathrm{mol}$ at $\left(340^{\circ}, 260^{\circ}\right)$ for $x=2$.

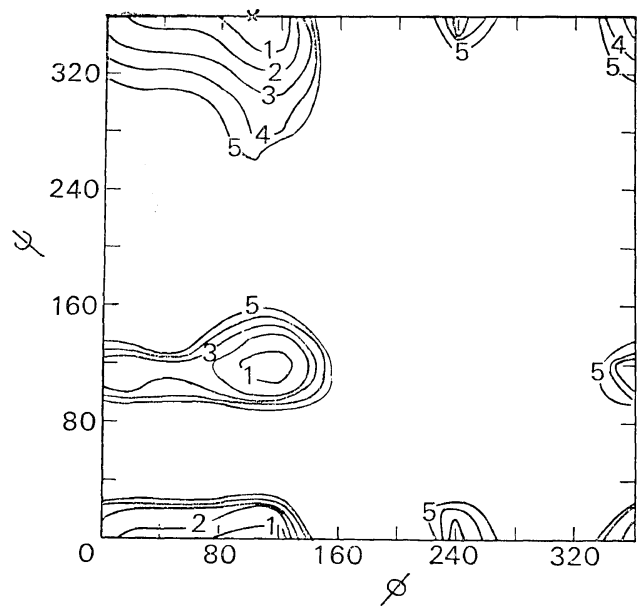

Figure 7. Contour map of total conformational energy for polypeptide with acidic side chains of $x=0$, calculated from eq 1 and 3 . The lowest energy point (the absolute energy value is -7.03 $\mathrm{kcal} / \mathrm{mol})$ is indicated by $\times\left(\right.$ at $\left.\varphi=100^{\circ}, \psi=360^{\circ}\right)$.

the total conformational energy contour maps are shown in Figures 7, 8, and 9. Figures 7, 8, and 9 are, respectively, obtained for acidic side chains with $x=0$, basic side chains with $x=1$, and basic side chains with $x=6$. Energy calculations were carried out at intervals of $10^{\circ}$ of $\varphi$ and $\psi$. Energy contours are given at intervals of $1 \mathrm{kcal} / \mathrm{mol}$ relative to the lowest energy value

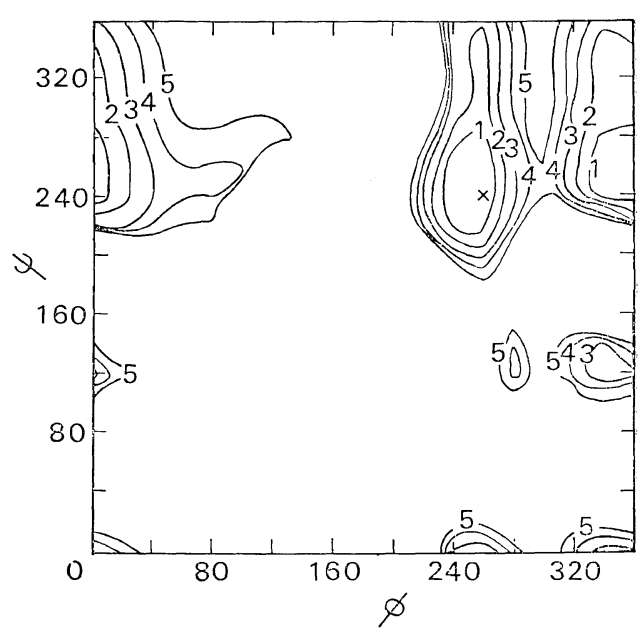

Figure 8. Contour map of total conformational energy for polypeptide with basic side chains of $x=1$, calculated from eq 1 and 3 . The lowest energy point (the absolute energy value is -13.8 $\mathrm{kcal} / \mathrm{mol})$ is shown by $\times\left(\right.$ at $\left.\varphi=260^{\circ}, \phi=240^{\circ}\right)$.

(shown by $x$ in the Figure). The calculated energy value and location $(\varphi, \psi)$ of the minimum point in each of the regions I, II, and III, for polypeptides having acidic and basic side chains with $x=0-6$, are summarized in the first to the third lines of Tables II and III. As is obvious from these Tables, the location of the minimum energy point is independent of $x$ in the regions 


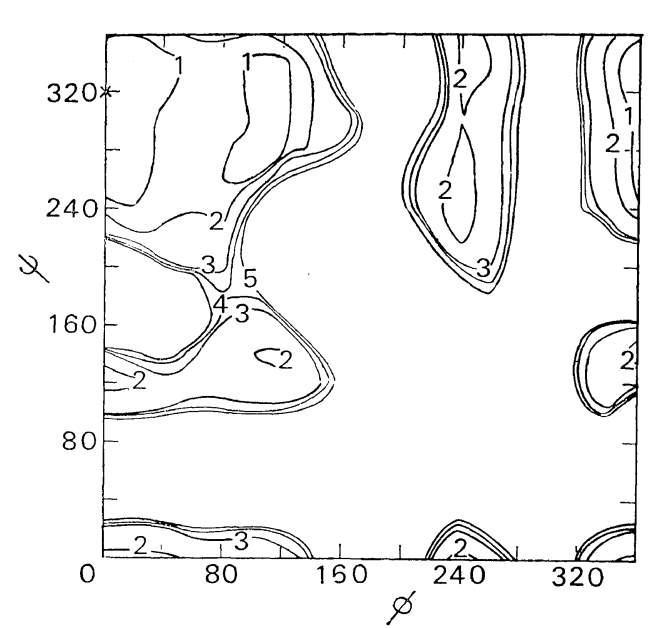

Figure 9. Contour map of total conformational energy for polypeptide with basic side chains of $x=6$, calculated from eq 1 and 3 . The lowest energy point (the absolute energy value is -3.98 $\mathrm{kcal} / \mathrm{mol}$ ) is indicated by $\times\left(\right.$ at $\left.\phi=0^{\circ}, \phi=32,0^{\circ}\right)$.

I and II, but dependent on $x$ in the region III. Furthermore, the lowest energy point in the contour maps is always located in region III except for the basic side chains with $x=0,1$, and 2.

\section{Conformational Entropy}

Owing to the planar trans conformation of the amide group, the conformational partition function of a polypeptide chain composed of $n$ residues is given by the $n$-th power of the partition function for each residue. The effect of amino acid side chains on the free energy of helix-to-coil transitions has been investigated by G. Némethy, et al. ${ }^{17}$ To evaluate the entropy of unfolding, they divided the $\varphi-\psi$ diagram into various regions which are sterically possible, and performed calculations by assuming a uniformly flat energy surface for each region. In the present paper, the entropy of the chain in the random-coil state was obtained by performing a statistical mechanical averaging over the entire range of rotational angles, $\varphi$ and $\phi$, taking into account the energies discussed in the preceding section.

The average conformational enery, $\langle V\rangle$, and the entropy, $S$, of the chain in the random-coil state were estimated from the following equations proposed by Brant, et al. ${ }^{12}$

$$
\langle V\rangle=z^{-1} \int^{2 \pi} \int_{0}^{2 \pi} V(\varphi, \psi) \exp [-V(\varphi, \psi) / R T] \mathrm{d} \varphi \mathrm{d} \psi
$$

In this equation, $z$ is the partition function per residue, and $V(\varphi, \psi)$ is the conformational energy given in eq 1. From another expression, $\langle V\rangle$ is equal to $R T^{2}(\mathrm{~d} \ln z / \mathrm{d} T)$. Accordingly, the entropy $S$ is given by eq 8 .

$$
S=R \ln z+R T \frac{\mathrm{d} \ln z}{\mathrm{~d} T}
$$

In practical calculations, the integral was replaced by the summation. The intervals of $\varphi$ and $\psi$ were $20^{\circ}$, and the assumed temperature was $25^{\circ} \mathrm{C}$.

The numerical values of $\langle V\rangle$ and $S$ calculated for poly(L-alanine) and polyglycine are given in Table IV. Also shown in this Table are the contributions of the torsional energy $\left\langle V_{(\mathrm{T})}\right\rangle$, the nonbonded van der Waals energy $\left\langle V_{(A A)}\right\rangle$, and the amide dipole-dipole interaction energy $\left\langle V_{(\mathrm{DD})}\right\rangle$, to the total mean energy $\langle V\rangle ;\langle V\rangle$ $=\left\langle V_{\langle\mathrm{T}\rangle}\right\rangle+\left\langle V_{(\mathrm{AA})}\right\rangle+\left\langle V_{\left\langle\mathrm{DD}_{1}\right\rangle}\right\rangle$. The results of calculation for polypeptides with ionized side chains of different $x$ are given in Table $\mathrm{V}$ for acidic side chain polymers, and in Table VI for basic ones. In these Tables, there are summarized the average total energy $\langle V\rangle$ and its five components (i.e., $\left.\left\langle V_{\langle\mathrm{T})}\right\rangle, V_{(\mathrm{AA})}\right\rangle,\left\langle V_{(\mathrm{DD})}\right\rangle$, $\left\langle V_{(\mathrm{DI})}\right\rangle$ and $\left\langle V_{(A \mathrm{I})}\right\rangle$ ), together with the conformational entropy $S$ calculated from the energies obtained by eq 1 and 3 . The quantities denoted by an asterisk (i.e., $\langle V\rangle^{*},\left\langle V_{(\mathrm{T})}\right\rangle^{*}, S^{*}$, etc.) were obtained by ignoring the induced electrostatic energy term in eq 3 .

It is meaningless to compare the absolute value of the entropy $S$ in the random-coil state calculated by eq 8 with that obtained from

Table IV. Average conformational energy, $\langle V\rangle$, its component energies, and entropy, $S$, of the chain in random-coil state, for polyglycine and polyalanine

\begin{tabular}{cccc}
\hline \multicolumn{2}{c}{ Parameter } & Poly-L-alanine & Polyglycine \\
\hline$\left\langle V_{(\mathrm{T})}\right\rangle$, & $\mathrm{kcal} / \mathrm{mol}$ & 0.90 & 0.81 \\
$\left\langle V_{(\mathrm{AA})}\right\rangle, \mathrm{kcal} / \mathrm{mol}$ & -1.69 & -2.53 \\
$\left\langle V_{(\mathrm{DD})}\right\rangle, \mathrm{kcal} / \mathrm{mol}$ & -0.75 & -0.67 \\
$\langle V\rangle$, & $\mathrm{kcal} / \mathrm{mol}$ & -1.54 & -2.39 \\
$S$, & $\mathrm{cal} / \mathrm{deg} \mathrm{mol}$ & 7.49 & 8.91 \\
\hline
\end{tabular}




\section{S. TANAKA and A. NAKAJIMA}

Table V. Average conformational energy, $\langle V\rangle$, its component energies, and entropy, $S$, for polypeptides with $-\left(\mathrm{CH}_{2}\right)_{x}-\mathrm{COO}^{-}$-type side chains

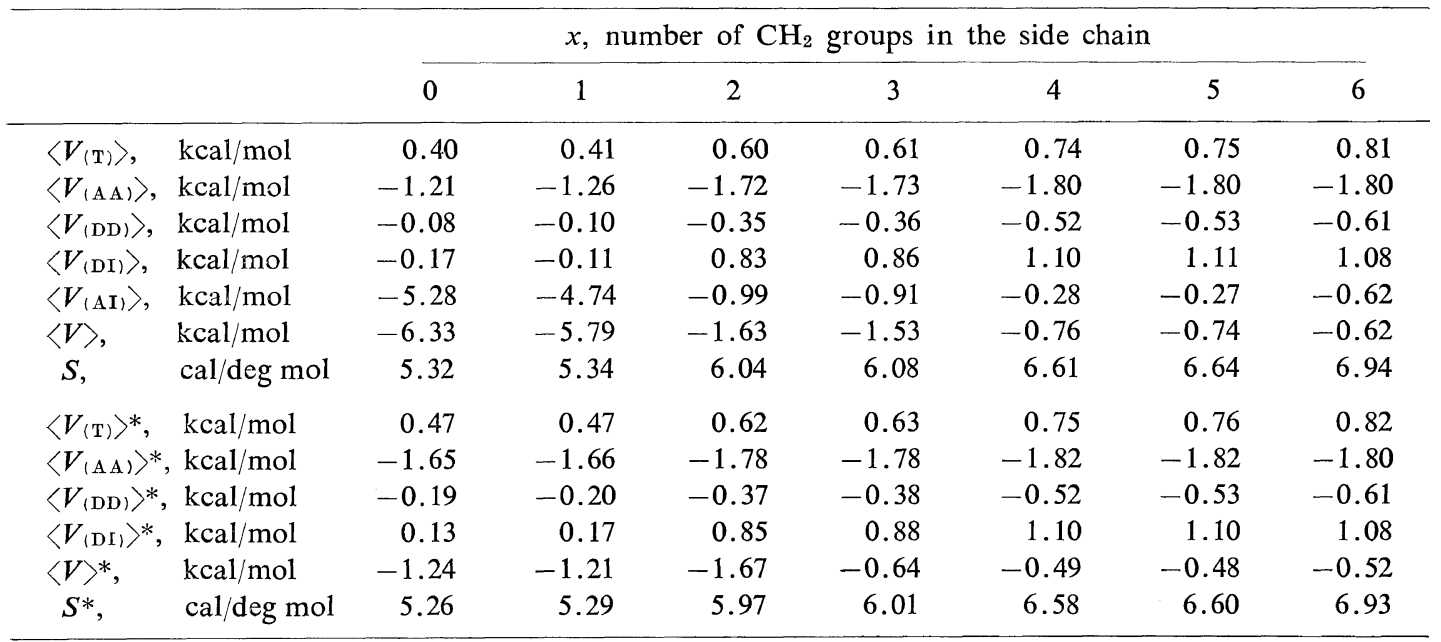

Table VI. Average conformational energy, $\langle V\rangle$, its component energies, and entropy, $S$, for polypeptides with $-\left(\mathrm{CH}_{2}\right)_{x}-\mathrm{NH}_{3}{ }^{+}$-type side chains

\begin{tabular}{|c|c|c|c|c|c|c|c|c|}
\hline & & \multicolumn{7}{|c|}{$x$, number of $\mathrm{CH}_{2}$ groups in the side chain } \\
\hline & & 0 & 1 & 2 & 3 & 4 & 4 & 6 \\
\hline$\left\langle V_{(\mathrm{T})}\right\rangle$, & $\mathrm{kcal} / \mathrm{mol}$ & 0.48 & 0.50 & 0.56 & 0.62 & 0.78 & 0.82 & 0.88 \\
\hline$\left\langle V_{(\mathrm{AA})}\right\rangle$ & $\mathrm{kcal} / \mathrm{mol}$ & 0.21 & 0.20 & -0.63 & -1.86 & -1.22 & -1.30 & -1.44 \\
\hline$\left\langle V_{(\mathrm{DD})}\right\rangle$ & $\mathrm{kcal} / \mathrm{mol}$ & 0.29 & 0.30 & -0.05 & -0.22 & -0.55 & -0.62 & -0.72 \\
\hline$\left\langle V_{(\mathrm{DI})}\right\rangle$ & $\mathrm{kcal} / \mathrm{mol}$ & -7.35 & -7.48 & -5.12 & -4.29 & -2.77 & -2.42 & -1.80 \\
\hline$\left\langle V_{(\mathrm{AI})}\right\rangle$, & $\mathrm{kcal} / \mathrm{mol}$ & -10.1 & -6.77 & -1.93 & -1.25 & -0.46 & -0.33 & -0.15 \\
\hline$\langle V\rangle$ & $\mathrm{kcal} / \mathrm{mol}$ & -16.5 & -13.3 & -7.18 & -5.99 & -4.21 & -3.85 & -3.23 \\
\hline$S$ & $\mathrm{cal} / \mathrm{deg} \mathrm{mol}$ & 6.05 & 6.04 & 6.83 & 7.07 & 7.49 & 7.57 & 7.66 \\
\hline$\left\langle V_{(\mathrm{T})}\right\rangle^{*}$ & $\mathrm{kcal} / \mathrm{mol}$ & 0.48 & 0.48 & 0.62 & 0.68 & 0.81 & 0.84 & 0.89 \\
\hline$\left\langle V_{(\mathrm{AA})}\right\rangle^{*}$, & $\mathrm{kcal} / \mathrm{mol}$ & -0.69 & -0.63 & -0.88 & -1.00 & -1.26 & -1.33 & -1.45 \\
\hline$\left\langle V_{(\mathrm{DD})}\right\rangle^{*}$, & $\mathrm{kcal} / \mathrm{mol}$ & 0.07 & 0.09 & -0.16 & -0.29 & -0.57 & -0.63 & -0.72 \\
\hline$\left\langle V_{(\mathrm{DI})}\right\rangle^{*}$ & $\mathrm{kcal} / \mathrm{mol}$ & -6.67 & -6.73 & -4.87 & -4.13 & -2.73 & -2.39 & -1.79 \\
\hline$\langle V\rangle^{*}$ & $\mathrm{kcal} / \mathrm{mol}$ & -6.81 & -6.79 & -5.29 & -4.75 & -3.75 & -3.51 & -3.08 \\
\hline$S^{*}$ & $\mathrm{cal} / \mathrm{deg} \mathrm{mol}$ & 6.12 & 6.17 & 6.86 & 7.11 & 7.52 & 7.59 & 7.67 \\
\hline
\end{tabular}

other methods, because the value of $S$ varies with the size of the intervals of $\varphi$ and $\psi$. To estimate the entropy of unfolding in the helixto-coil transition from the above-mentioned $S$, the entropy of the chain in the helix conformation should be necessary. In this work, however, we will not be concerned with the entropy of unfolding. Our aim is to compare the degrees of the destabilization of helices, with regard to polypeptides having ionizable side chains of different $x$. Therefore relative comparison is sufficient for this purpose. The difference in $S$ for poly(L-alanine) and polyglycine is -1.42 cal/deg mol ( $c f$. Table IV). This means that the helix form of polyalanine is entropically more stable than that of polyglycine. Némethy, et $a l .{ }^{17}$, have reported a similar result, though their value $-2.4 \mathrm{cal} / \mathrm{deg} \mathrm{mol}$ is somewhat larger than ours. They have discussed further the effect of side chains on the entropy of polypeptides in the random-coil form relative to the contribution from the backbone, by taking into 


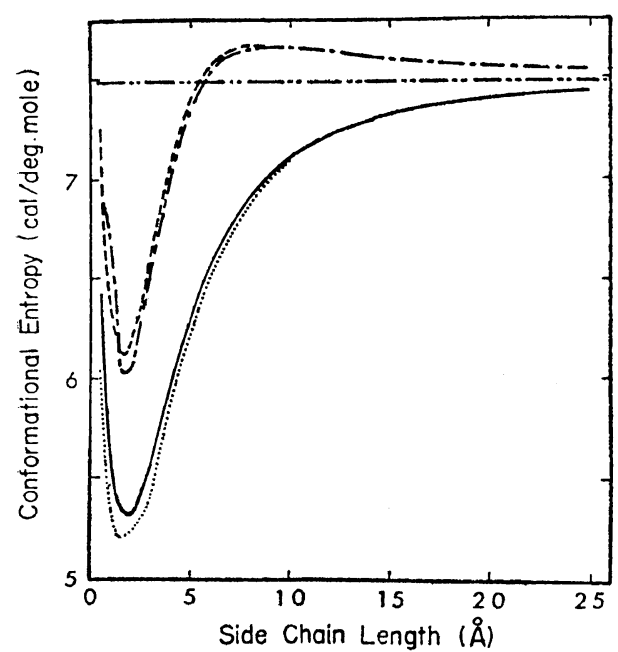

Figure 10. Dependence of conformational entropy in the random-coil state on the side-chain length given by the distance $(\AA)$ between the atom $C^{\alpha}$ and the ionic charge $I:-$, from eq 1 and 3 , assuming that $V_{(\mathrm{AI}) j k}(\varphi, \phi)=0$, for acidic sidechain polymers; $\cdots \cdots$, from eq 1 and 3 , for acidic side-chain polymers; - - - - - from eq 1 and 3 , assuming that $V_{\left(\mathrm{A}_{\mathrm{I}}\right) j k}(\varphi, \phi)=0$, for basic side-chain polymers; --.--, from eq 1 and 3 , for basic side-chain polymers; - -....., for un-ionized polymers.

account only nonbonded atom interaction energies, and pointed out that the conformational energy of $\alpha$-L-polypeptides with unbranched side chains including more $\mathrm{C}$ atoms than $\mathrm{C}^{r}$ atoms is almost the same as that of polyalanine, in spite of the greater effect of branched side chains.

Figure 10 shows the conformational entropy $S$ in the random-coil form as a function of the side-chain length, the distance from $\alpha$-carbon to the ionic charge being projected in the $\mathrm{C}^{\alpha}-\mathrm{C}^{\beta}$ direction. As mentioned previously, the value of $S$ for $\alpha$-L-polypeptides with un-ionized side chains is constant, $S=7.49 \mathrm{cal} / \mathrm{deg} \mathrm{mol}$, independent of the chain length. As is obvious from Figure 10, with increasing chain length (i.e., the distauce between the $\mathrm{C}^{\alpha}$ and point I in Figure 1a), the effect of ionic charges is reduced and the entropy value approaches $S=$ $7.49 \mathrm{cal} / \mathrm{deg} \mathrm{mol}$ which is assigned for unionized chains. This method of approach is affected by the dielectric constant $\varepsilon$ of the sur- rounding medium and/or the screening effect of added small electrolytes. As can be seen from Figure 10 and Tables V and VI, there is no remarkable difference between the conformational entropies calculated either by taking into account the contribution from induced electrostatic interaction energy or not doing so. Practical differences are not observed at all for side chains longer than $10 \AA$. The entropy curves for acidic and basic side chains, respectively, pass through a minimum and increase with the side-chain length, but both curves are different in that, for acidic side chain, $S$ increases monotonically to the limiting value 7.49 with the chain length, and therefore, is always smaller than 7.49. For the basic side chain, the curve reaches a maximum at about $8 \AA$ and then approaches 7.49 from another direction, i.e., in this region the entropy values are sightly higher than those for un-ionized side chains (see Figure 10). The entropy difference in the random-coil state between ionized and unionized side-chain forms is $-2.2 \mathrm{cal} / \mathrm{deg} \mathrm{mol}$ for PLAA and $-1.5 \mathrm{cal} / \mathrm{deg} \mathrm{mol}$ for PLGA, as seen in Table V. Comparison of PLAA or PLGA, both in ionized form, with polyglycines (PG) results in an entropy difference of $-3.6 \mathrm{cal} / \mathrm{deg}$ mol for the former and $-2.9 \mathrm{cal} / \mathrm{deg} \mathrm{mol}$ for the latter. These values are larger in absolute value than the entropy difference $-1.42 \mathrm{cal} / \mathrm{deg}$ mol between un-ionized PLAA or PLGA, and PG. These results suggest that, in the case of acidic side chains, the conformational entropy (absolute value) in the random-coil form is reduced by ionization and thus the ionized helix form becomes entropically more stable than un-ionized helix form. Furthermore, the region I (Figure 4) mainly contributes to the entropy in the random-coil state. With respect to the basic side chains, of small $x$, the regions II and III mainly contribute to the random-coil entropy (Figure 8); in other words, the right-handed $\alpha$-helix form becomes energetically unstable because of the high energy level in region I. For large $x$ (see Figure 9), region III is mainly associated with the randomcoil entropy, which, moreover, is larger than that of the un-ionized state, though only slightly.

In what follows, we will discuss the pHinduced helix-coil transition for polypeptides 


\section{S. TANAKA and A. NAKaJima}

with ionizable side chains, in comparison with experimental results hitherto reported. Previously mentioned theoretical considerations may apply to the early stage of the helix-to-coil transition. We now consider an initial stage of helix-to-coil (not coil-to-helix) transition, in which ionized groups are few in number and therefore are isolated from un-ionized groups. In the case of polypeptides with acidic side chains, especially for small $x$ (i.e., PLAA, PLGA), the random-coil entropy $S$ is reduced by ionizaton; this means that the helix conformation is entropically more favorable and ionized helix may be realized at this stage. Accordingly, some other interactions are necessary to promote the helix-to-coil transition. For example, the long-range Coulombic interactions among an ionized carboxyl group and its neighboring ionized carboxyl groups may play a major role. At a degree of ionization, where these long-range interactions overcome the total effect due to the decrease in the random-coil entropy and the energy of intramolecular hydrogen bonds, an abrupt helix-to-coil transition may occur. On the basis of the results obtained by Wada $^{3}$ for $\mathrm{pH}$-induced helix-to-coil transition of PLGA, it has been pointed out ${ }^{22}$ that a rather high degree of ionization is necessary to promote helix-to-coil transition.

On the other hand, for polypeptides with basic side chains, when a $-\mathrm{NH}_{2}$ group is ionized, the helix-to-coil transition may start immediately from this residue. After ionization has taken place to some extent, the transition may proceed at accelerated pace with the aid of the interaction between ionized side chain charges.

Peller $^{23}$, in his theoretical consideration, gave the degree of ionization, $\alpha$, for molecules entrusted to the conformational transition, as an average of the degrees of ionization for the helix, $\alpha_{\mathrm{h}}$, and for the coil $\alpha_{\mathrm{e}}$.

$$
\alpha=\theta \alpha_{\mathrm{h}}+(1-\theta) \alpha_{\mathrm{c}}
$$

In eq $9, \theta$ denotes the fraction of monomer units having intramolecular hydrogen bonds. The titration curve for the helix-to-coil transition approaches that for a charged helix at the $\mathrm{pH}$ region below the transition point, while it approaches that for random coil at the $\mathrm{pH}$ region above the transition point. Such a supposition may be reasonable for polyacids, from both the energy and entropy points of view. On the other hand, the titration curve for polyL-lysine reported by Applequist and Doty ${ }^{4}$ was in the form $\alpha=\alpha_{\mathrm{c}}(1-\theta)$ at $\alpha<0.6$. In other words, ionization of the $-\mathrm{NH}_{2}$ groups of the side chains has not taken place in helix form $\left(\alpha_{\mathrm{h}}=0\right)$. This fact is in accord with our conclusion that the helix conformation is unstable, energetically and entropically, when the side chains are ionized. This result suggests that the equation $\alpha=\alpha_{\mathrm{c}}(1-\theta)$ is applicable even at $\theta \cong 1$. More detailed discussion on the helixcoil transition is outside of the scope of this report. Other problems such as the entropy for helix form; entropy contribution of the side chain itself; effect of hydrogen bonds; interaction of the side chain itself; effect of hydrogen bond; interaction of polymers with solvents and the screening effect of added salts must also be considered. Lastly, the results obtained in this paper by assuming $\varepsilon=3.5$ in the calculation of dipoledipole interaction energy and $\varepsilon=1$ in eq 4 may overestimate the charge effect in aqueous solution. The choice of these numerical values for the effective dielectric constant, $\varepsilon$, remains unsolved.

Computations were carried out on the KDC-II computer at Kyoto University Computation Center.

\section{REFERENCES}

1. P. Doty, A. Wada, J. T. Yang, and E. R. Blout, J. Polym. Sci., 23, 851 (1957).

2. P. Doty, K. Imahori, and E. Klemperer, Proc. Natl. Acad. Sci., 44, 424 (1958).

3. A. Wada, Mol. Phys., 3, 409 (1960).

4. J. Applequist and P. Doty, in "Polyamino Acids, Polypeptide and Protein," M. A. Stahmann, Ed., University of Wisconsine Press, Medison, Wisc. 1962, p 161.

5. Y.P. Myer, Macromolecules, 2, 624 (1969).

6. M. L. Tiffany and S. Krimm, Biopolymers, 6, 1379 (1968).

7. A. Ciferri, D. Puett, L. Rajagh and J. Hermans, Jr., Biopolymers, 6, 1019 (1968).

8. B. H. Zimm and S. A. Rice, Mol. Phys., 3, 391 (1960).

9. S. Krimm and J. E. Mark, Proc. Natl. Acad. Sci., 60, 1122 (1968).

10. L. Pauling, "The Nature of Chemical Bond," 
3rd ed, Cornell University Press, Ithaca, N.Y., 1960.

11. S. Tanaka and A. Nakajima, Polymer J., 1, 71 (1970).

12. D. A. Brant, W. G. Miller, and P. J. Flory, J. Mol. Biol., 23, 47 (1967).

13. D. A. Brant and P. J. Flory, J. Amer. Chem. Soc., 87, 2791 (1965).

14. R. A. Scott and H. A. Scheraga, J. Chem. Phys., 45, 2091 (1966).

15. T. Ooi, R. A. Scott, G. Vanderkooi, and H. A. Scheraga, ibid., 46, 4410 (1967).

16. For example, see J. C. Slater and N. H. Frank, "Introduction to Theoretical Physics,"' McGrawHill, New York and London, 1933.

17. G. Némethy, S. J. Leach, and H. H. Scheraga,
Phys. Chem., 70, 988 (1966).

18. S. J. Leach, G. Nèmethy, and H. A. Scheraga, Biopolymers, 4, 369 (1966).

19. C. Ramakrishnan and G. N. Ramachandran, Biophys. J., 5, 909 (1965).

20. G. N. Ramachandran, C. Ramakrishnan, and J. V. Sasisekharan, J. Mol. Biol., 7, 95 (1963).

21. S. Lifson and A. Roig, J. Chem. Phys., 34, 1963 (1961).

22. T. M. Birshtein and O. B. Ptitsyn, "Conformations of Macromolecules," S. N. Timasheff and M. J. Timasheff, Translators, Interscience Publishers, Inc., New York, N. Y., 1966, Chapter 10.

23. L. Peller, J. Phys. Chem., 63, 1194 (1959). 